SCHWEIZERISCHE ZEITSCHRIFT FÜR HYDROLOGIE BIRKHÄUSER VERLAG BASEL

Eidg. Technische Hochschule, Zürich - Fortbildungskurs der EAWAG, 1964

\title{
Möglichkeiten des Komposteinsatzes im Pflanzenbau
}

Von P. Keller, Eawag, Zürich 


\section{Einleitung}

Für die Verfahrenswahl bei der Projektierung einer Abfallbeseitigungsanlage spielt die Frage, ob Kompost in der näheren Umgebung abgesetzt werden kann oder nicht, eine wesentliche Rolle. Ferner ist bei Kompostwerken, die neu in Betrieb genommen wurden, mit einer gewissen Anlaufszeit zu rechnen, bis sich der Kompostabsatz eingespielt hat; während dieser Zeit ist eine gezielte Werbung notwendig, und es ist daher für den Betriebsleiter wichtig, zu wissen, wer als potentieller Abnehmer in erster Linie in Frage kommt.

Als Kompostabnehmer kommen diejenigen Richtungen des Pflanzenbaus in Betracht, die durch die Anwendung von Kompost einen betrieblichen Vorteil irgendwelcher Art, d. h. letzten Endes einen wirtschaftlichen Nutzeffekt erwarten können. Die Beantwortung der Frage, ob in einem bestimmten Fall die Anwendung von Kompost tatsächlich wirtschaftlich ist oder nicht, ist jedoch nicht immer einfach, da sich folgende Schwierigkeiten bei der Beurteilung der Kompostwirkung zeigen:

Kompost ist ein Bodenverbesserungsmittel (Bodendünger); er wirkt in erster Linie indirekt auf die Pflanze, indem er verschiedene sog. Milieufaktoren verändert, die dann wiederum das Wachstum der Pflanze beeinflussen. Die Direktwirkung des Kompostes - durch Nährstoffe, Spurenelemente usw. - ist dagegen sekundär, im Gegensatz zu den eigentlichen Pflanzendüngern, z. B. den Mineraldüngern. Es ist daher verständlich, dass die Beziehungen zwischen Aufwand und Erfolg bei der Kompostanwendung viel weniger linear sind als bei den Pflanzendüngern. Dazu kommt noch die meist grössere Anzahl Faktoren, die bei der Beurteilung der Kompostwirkung zu berücksichtigen sind.

Der Kompost bewirkt Veränderungen einiger physikalischer und chemischer Bodeneigenschaften; diese Veränderungen gehen in der Regel nur 
allmählich vor sich und erstrecken sich über einen grösseren Zeitraum. So wird z. B. ein optimaler Wasserhaushalt in einem bestimmten Boden erst nach mehrjähriger Kompostanwendung erreicht werden können, und zudem ist die Wirkung der erreichten Verbesserung des Wasserhaushaltes unter extremen Bedingungen (sehr nass oder sehr trocken) natürlich am grössten. Es bedarf daher u. U. einer mehrjährigen Versuchsfolge, um die Kompostwirkung in einem bestimmten Fall richtig beurteilen zu können.

Kompost aus Siedlungsabtällen ist in erster Linie vergleichbar mit den bereits bekannten Bodendüngern, wie Gartenkompost, Stallmist, Torf usw. Vielfach werden diese Stoffe in der Praxis mehr nach Tradition und Gefühl angewendet als auf Grund wirtschaftlicher Überlegungen, oder sie werden, sofern sie auf dem Betriebe selbst anfallen, als mehr oder weniger notwendiges Übel betrachtet. Es ist daher nicht verwunderlich, dass auch der Abfallkompost in der Praxis sehr verschieden beurteilt wird, je nach dem jeweiligen subjektiven Standpunkt, zumal objektive Untersuchungen und Berechnungen noch weitgehend fehlen.

Das ganze Gebiet des Humushaushaltes und der Humuswirkung im Boden hat erst in jüngerer Zeit vermehrte Beachtung durch die Fachwelt gefunden. Aus diesem Grunde, und ebenfalls wegen der Komplexheit der dabei auftretenden Probleme sowie der methodischen Schwierigkeiten bei der Abklärung einzelner Fragen, ist es verständlich, dass die Ergebnisse der Forschung nur zu einem kleinen Teil auf die Praxis übertragen werden konnten. Dies führt dazu, dass gerade hier sehr leicht Behauptungen aufgestellt, aber nur schwer'Tatsachen bewiesen werden können. Dazu kommt noch die Verwirrung, die angestiftet wird durch mehr weltanschaulich bedingte Meinungen und Ansichten, vor allem aus sog. «biologischen» Kreisen, sowie die entsprechende, auch nicht immer objektive Gegenreaktion der Fachwelt.

Man kann es immer wieder erleben, dass sogar bei den von Fachleuten zur Feststellung der Kompostwirkung durchgeführten Versuchen bereits die Versuchsanlage durch Erwägungen dogmatischer Art bestimmt und daher die Versuchsergebnisse entsprechend diskutabel sind. Auch bei der Mitteilung von Ergebnissen aus der Praxis hält es manchmal schwer, Tatsachen von Behauptungen zu trennen.

Es besteht bereits eine umfangreiche Literatur mehr theoretischer Art über die Bedeutung und die Wirkung vom Kompost, mit der leider aus den obenerwähnten Gründen der Praktiker im allgemeinen nicht sehr viel anfangen kann. Wir möchten daher hier nicht ebenfalls von theoretischen Überlegungen ausgehen, sondern vielmehr rein empirisch die bisherigen 
praktischen Erfahrungen bei der Kompostanwendung zusammenstellen. Dabei sollen diejenigen Richtungen des Pflanzenbaus herausgestellt werden, für die die Anwendung von Abfallkompost auch in Zukunft zweckmässig und wirtschaftlich erscheint.

\section{Die Wirkung des Kompostes auf Boden und Pflanze}

Die Bedeutung des Kompostes liegt darin, dass dem Boden organische Stoffe in mehr oder weniger humifizierter Form zugeführt werden. Durch die Anwendung von Kompost kann also der Humusgehalt des Bodens entweder erhöht oder, da Humus ständig abgebaut und daher wieder ersetzt werden muss, doch auf dem bestehenden Niveau erhalten werden.

Daneben enthält der Kompost noch Pflanzennährstoffe (N, P, K) sowie Spurenelemente und andere Mineralstoffe. Der Nährstoffgehalt ist gering, vor allem bei reinen Müllkomposten, und zudem ist der grösste Teil dieser Nährstoffe nicht sofort pflanzenverfügbar; rein als Dünger betrachtet ist daher Kompost im Vergleich zu den Mineraldüngern unwirtschaftlich. Über die Bedeutung der Spurenelemente im Kompost sind vorläufig nur sehr wenig Tatsachen bekannt, weshalb diese Frage aus den weiteren Betrachtungen weggelassen werden soll.

Die Tatsache, dass der Kompost in erster Linie ein Humuslieferant, also ein Bodendünger ist, bedeutet, dass die Anwendung von Kompost vor allem dort in Frage kommt, wo man dem Humushaushalt im Boden besondere Aufmerksamkeit schenken muss. Da in der Schweiz die landwirtschaftlich genutzten Böden, bedingt durch das günstige Klima, die hohe Stallmistproduktion sowie eine geeignete Anbaufolge (Fruchtwechsel) genügend mit Humus versorgt sind, kommt eine Anwendung von Kompost hier nicht in Frage. Diese Überlegung gilt im Prinzip auch für die anderen westeuropäischen Länder; auch dort, wo extreme Bodenverhältnisse an sich den Einsatz von Kompost rechtfertigen würden, wird man aus wirtschaftlichen Gründen eher mit Hilfe anderer Massnahmen - z. B. Gründüngung, Strohmulch usw. - den Humusgehalt zu verbessern suchen. Es ist zudem damit zu rechnen, dass im Zuge der zunehmenden Konzentrationsbestrebungen innerhalb der europäischen Landwirtschaft die extremen Standorte mit der Zeit aufgegeben werden.

Es zeigt sich immer mehr, dass der Zukauf organischer Düngemittel nur dort wirtschaftlich sein kann, wo pro Flächeneinheit quantitativ und qualitativ sehr hohe Erträge erzielt werden, also im Intensivbau. Auch hier können jedoch keine allgemeingültigen Richtlinien herausgearbeitet werden, 
sondern jeder Betrieb muss für sich selbst, auf Grund seiner besonderen Verhältnisse herausfinden, ob die Verwendung organischer Bodendünger gerechtfertigt ist oder nicht. Um zu beurteilen, ob sich insbesondere die Verwendung von Kompost aus Siedlungsabfällen lohnt oder nicht, muss einmal der Gestehungspreis, inkl. Transport und Ausbringung, im Vergleich zu ähnlichen Produkten wie Stallmist und Torf untersucht werden, und ferner eine klare Vorstellung über die zu erwartende Wirkung vorhanden sein.

Die Humusstoffe, zu deren Vermehrung und Erhaltung der Kompost angewandt wird, beeinflussen den chemischen und physikalischen Aufbau des Bodens in folgendem Sinne:

Die Humusstoffe sind wesentlich an der Bildung einer stabilen, lockeren Krümelstruktur des Bodens beteiligt. Ein krümeliger Boden hat ein grösseres Porenvolumen, was den Gasaustausch mit der Atmosphäre und infolgedessen die Wurzelatmung verbessert, und zudem eine bessere Dränagefähigkeit. Krümeliger Boden ist leichter zu bearbeiten und ermöglicht eine bessere Durchwurzelung durch die Pflanzen.

Die Humusstoffe erhöhen die Wasserkapazität des Bodens, so dass er in Trockenzeiten weniger schnell austrocknet und nicht verhärtet.

Die Humusstoffe sind Ionenaustauscher, die die adsorbierten Nährstoffe langsam und gleichmässig an die Pflanze abgeben; dadurch wird eine Schockwirkung unmittelbar nach der Düngung vermieden und zugleich werden die Nährstoffverluste durch Auswaschung herabgesetzt.

In Steilhanglagen ohne Bodenbedeckung, wie sie vor allem im Weinbau die Regel sind, verhindern die Humusstoffe die Erosion der Feinerde nach starken Niederschlägen, indem die mineralischen Feinstteile zu stabilen Krümeln zusammengebunden werden, und ferner durch die Verbesserung der Dränagefähigkeit ein grösserer Teil der Niederschläge im Boden versickern kann.

Die Humusstoffe bilden das Nährsubstrat für die ganze Bodenlebewelt, die für die Bodenfruchtbarkeit von grösster Bedeutung ist. Die bei Monokulturen häufige Bodenmüdigkeit infolge der Bildung einseitiger und schädlicher Populationen kann durch die Erhaltung eines genügenden Humusgehaltes bekämpft werden.

Aus dieser Zusammenstellung geht hervor, dass den Humusstoffen in erster Linie eine Pufferwirkung zukommt, d. h. ihre Wirkung liegt im wesentlichen darin, dass sie zu der Schaffung eines ausgeglichenen Milieus für die Pflanze beitragen und so mithelfen, extreme Stösse von irgendeiner Seite aufzufangen. Tatsächlich kann auch festgestellt werden, dass die Er- 
träge auf gut mit Humus versorgten Böden im Durchschnitt ausgeglichener sind als auf Böden mit niedrigem Humusgehalt. Als Erfolg z. B. der Anwendung von Kompost braucht durchaus nicht immer eine Ertragssteigerung einzutreten, sondern gerade im Intensivbau kann die Erhaltung der Bodenfruchtbarkeit sowie die Verhütung von ausgesprochenen Fehlerträgen infolge schlechter Witterung u. ä. wirtschaftlich interessant sein. In einigen Fällen will man mit dem Kompost auch gar nicht den Ertrag beeinflussen, sondern eher den Aufwand, z. B. für die Bodenbearbeitung oder die Behebung von Erosionsschäden, herabsetzen.

Infolge der steigenden Ansprüche der Konsumenten spielt in den letzten Jahren bei vielen Produkten des Obst- und Gartenbaus die Qualität eine mindestens ebenso grosse Rolle wie die Quantität; es kann wirtschaftlicher sein, in kleineren Mengen Qualitätsprodukte zu erzeugen als grosse Mengen zweiter Qualität. Gerade in qualitativer Hinsicht spielen Fragen der Bodenfruchtbarkeit, der Humusversorgung usw. bei vielen Produkten eine entscheidende Rolle, und es muss daher neben der reinen Pflanzendüngung auch den Massnahmen zur Verbesserung und Erhaltung einer guten Bodenstruktur genügend Aufmerksamkeit geschenkt werden.

Tabelle 1

Vergleich der Zusammensetzung pon Mül-Klärscblammkompost, Torf und Stallmist

Die \%-Zahlen beziehen sich auf das ursprüngliche, feuchte Material; es handelt sich dabei um Durchschnittswerte aus sehr vielen Einzelanalysen

\begin{tabular}{llll}
\hline & $\begin{array}{l}\text { Müll-Klärschlamm- } \\
\text { kompost (ausgereift) } \\
\%\end{array}$ & Torf & \multicolumn{2}{l}{ Stallmist } \\
& $\%$ & $\%$ & $\%$ \\
\hline Wassergehalt & $40-50$ & etwa 30 & $75-80$ \\
Organische Substanz & $20-40$ & etwa 60 & 17 \\
Stickstoff $(\mathrm{N})$ & $0,3-0,9$ & $0,5-1$ & 0,5 \\
Phosphor $\left(\mathrm{P}_{2} \mathrm{O}_{5}\right)$ & $0,3-0,9$ & - & 0,3 \\
Kali $\left(\mathrm{K}_{2} \mathrm{O}\right)$ & $0,3-0,6$ & - & 0,6 \\
Magnesium $(\mathrm{MgO})$ & $0,3-0,9$ & - & - \\
Kalzium $(\mathrm{CaO})$ & $0,6-6$ & - & - \\
\hline
\end{tabular}

\section{Die Entwicklung des Kompostabsatzes in den letzten Jahrzehnten}

Die Herstellung von Kompost aus Siedlungsabfällen war früher vor allem darauf ausgerichtet, ein Bodenverbesserungsmittel für die Rekultivierung von Ödland zu gewinnen. So wurde z. B. das grosse Kompostwerk Wijster in den Niederlanden anfangs der dreissiger Jahre ausschliesslich zu dem Zwecke erstellt, Humus für die Melioration der Sandböden im Osten des Landes zu erhalten; aus ähnlichen Gründen wurde auch der Müll der Stadt 
Bern zur Urbarmachung der Flugsandböden der Strafanstalt Witzwil im Seeland verwendet.

Seitdem nun das Interesse der einzelnen Staaten Westeuropas, ohne Rücksicht auf wirtschaftliche Erwägungen in bezug auf die Landwirtschaft möglichst autark zu sein, immer mehr abnimmt, ist auch die Frage der Erschliessung und landwirtschaftlichen Nutzung extremer Böden nicht mehr aktuell. Es muss im Gegenteil damit gerechnet werden, dass sich die Landwirtschaft immer mehr auf diejenigen Standorte, bzw. Böden, konzentriert, die auch ohne kostspielige Meliorations- und Bodenverbesserungsmassnahmen fruchtbar sind, und dass die extremen Standorte aufgegeben werden. Wohl muss auch die Landwirtschaft die Humusversorgung und die Erhaltung der Bodenfruchtbarkeit vermehrt beachten, aber dies wird in erster Linie durch geeignete betriebsinterne Massnahmen zu geschehen haben und nicht durch Ankauf relativ teurer, organischer Bodendünger.

Wie wir bereits erwähnt haben, ist heute vor allem der Intensivbau der potentielle Abnehmer für organische Bodendünger; diese Tendenz dürfte sich in Zukunft noch verstärken. Dass insbesondere der Kompost aus Siedlungsabfällen bei der Humusversorgung der Intensivbetriebe eine gewisse Rolle spielen kann, hat folgende Gründe:

Ein grosser Teil der Intensivbaubetriebe war schon von jeher auf den Ankauf organischer Bodendünger angewiesen, vor allem von Stallmist und Torf. Der Preis dieser beiden Produkte ist jedoch in den letzten Jahren sehr stark gestiegen. Hochmoortorf kostet heute, je nach Bezugsquelle, Fr. 200-300.- pro t, und für Stallmist werden Preise von Fr. 30-40.- pro t verlangt und auch bezahlt. Im Vergleich dazu ist der Preis für Müllkompost (Fr. IO-20.- pro t, inkl. Transport) sehr günstig.

Durch die Fortschritte der Düngung, Züchtung, Bodenbearbeitung, Schädlingsbekämpfung usw. ist gerade im Intensivbau der Ertrag quantitativ und qualitativ sehr stark gestiegen. Hochgezüchtete und gutgedüngte Pflanzen sind jedoch empfindlich, und es besteht ständig die Gefahr grösserer Ertragseinbussen bei ungünstigen Umweltbedingungen. In Anbetracht der hohen Summen, die jeweils auf dem Spiele stehen, ist die Schaffung und Erhaltung eines für die Pflanze günstigen, ausgeglichenen Milieus sowie die weitestmögliche Stabilisierung der Milieufaktoren von grösster Bedeutung (Extremfall: Gemüse- und Blumenzucht in geheizten Glashäusern).

Der Intensivbau verlangt sehr viel Handarbeit. Da auch hier die Arbeitslöhne sehr stark angestiegen sind, besteht ein Interesse an allen Massnahmen, die den Arbeitsaufwand herabsetzen. Durch die Verbesserung der 
Bodeneigenschaften mit Hilfe organischer Bodendünger kann in vielen Fällen der Aufwand für die Bodenbearbeitung oder für die Behebung von Erosionsschäden vermindert werden. Bei der Anwendung von Müllkompost kommt noch dazu, dass dieser viel leichter auszubringen ist als der Stallmist.

Für die Herstellung von Gartenkompost aus den betriebseigenen Abfällen ist ebenfalls viel Handarbeit notwendig, trotz weitgehender Mechanisierung; der Gartenkompost dürfte daher dem Gärtner wesentlich teurer zu stehen kommen als der zugekaufte Müllkompost. Da jedoch die betriebseigenen Abfälle auf irgendeine Art und Weise beseitigt werden müssen, wäre in gewissen Fällen zu untersuchen, ob nicht diese Abfälle in einem Kompostwerk aufbereitet und dafür Kompost bezogen werden könnte.

\section{Beurteilung der Möglichkeiten zur Anwendung von Kompost aus Siedlungsabfällen, unter spezieller Berücksichtigung der Verhältnisse in der Schweiz}

\section{I Landwirtschaft}

4. I I Acker- und Futterbau. Sofern Stallmist produziert wird, kann bei diesen Betrieben die Humusversorgung durch interne Massnahmen aufrechterhalten werden (s. oben); der Ankauf von organischen Bodendüngern ist in der Regel unwirtschaftlich.

In der letzten Zeit nimmt die Zahl der viehlosen Landwirtschaftsbetriebe stark zu. Es ist durchaus möglich, dass in gewissen Fällen die Humusversorgung durch Müllkompost wirtschaftlich interessant sein kann. Ansätze in dieser Richtung zeigen sich in Deutschland und den Niederlanden und sollten aufmerksam verfolgt werden.

4.I2 Viebhaltung. Mehr oder weniger reine Graswirtschaftsbetriebe sind in der Regel gezwungen, Einstreumaterial (Stroh, Torf) zuzukaufen; die Preise dafür sind in der letzten Zeit stark gestiegen. In den Niederlanden wird bereits seit Jahren in vielen Betrieben Kompost als Einstreu verwendet, und in der Region Zürcher Oberland sind Versuche im Gange, um diese Möglichkeit auch in bezug auf unsere Verhältnisse zu untersuchen.

Kompost ist wesentlich preisgünstiger als Stroh oder Torf, und zudem verrottet der mit Kompost vermischte Stallmist besser und schneller als reiner Strohmist. Das Endprodukt ist krümelig, was die Ausbringung erleichtert.

Sofern die in dieser Richtung durchgeführten Versuche positiv ausfallen, besteht die Möglichkeit, auch in Gebieten mit wenig Intensivbau, z. B. den Voralpen, grössere Kompostmengen abzusetzen. 
In vielen Fällen ist das Ausbringen von Gülle umständlich, und man würde lieber mehr Stallmist erzeugen. Beim Einstreu von Kompost in den Stall kann durch Ausnützung der hohen Wasserkapazität des Kompostes der Gülleanfall herabgesetzt und dafür die Stallmistproduktion (und -qualität) erhöht werden.

\subsection{Forstwirtschaft}

4.2I Pflanzennachzucht in Forstgärten. Der Bedarf an Jungpflanzen für die Verschulung nimmt stark zu und dürfte auch in Zukunft weiter zunehmen, da einmal der natürliche Nachwuchs in den intensiv genutzten Wäldern des Mittellandes immer weniger genügt und weil ferner schon in nächster Zukunft grössere Flächen vor allem im Voralpengebiet aufgeforstet werden dürften. Die Forstgartenfläche, die gegenwärtig ungefähr 300 ha beträgt, wird daher auf 400-500 ha zunehmen.

Die meisten Forstgärten sind zwangsläufig auf relativ schlechten Böden angelegt; dazu kommt, dass bei der Verschulung mit den Wurzelballen ein Teil der Oberflächenerde mitgenommen wird. Früher mussten daher die Forstgärten in der Regel alle IO-I2 Jahre verlegt werden, mit entsprechenden Kosten für die Neuanlage. Versuche, die an verschiedenen Orten von der Eidgenössischen Forstlichen Versuchsanstalt durchgeführt wurden, haben gezeigt, dass durch die Anwendung relativ grosser Kompostmengen nicht nur das Verlegen der Forstgärten überflüssig wird, sondern dass auch das Wachstum der Jungpflanzen verbessert und die Ertragsausfälle herabgesetzt werden können. Die Anwendung von Kompost verhindert auch die Verunkrautung und kann dadurch gewisse Verbesserungen in arbeitstechnischer Hinsicht bringen. Die Versuche haben ferner gezeigt, dass im Durchschnitt die Jungpflanzen I Jahr früher verschult werden können.

Aus all diesen Gründen gehören die Forstgärten zu den besten Kompostabnehmern.

4.22 Aufforstungen. Aufgeforstet werden vor allem diejenigen Böden, deren anderweitige Nutzung sich nicht mehr lohnt. In vielen Fällen, vor allem in Deutschland und in den Niederlanden, hat sich gezeigt, dass eine einmalige Bodenverbesserung durch Kompost zu guten Ergebnissen führt, ja sogar die Voraussetzung für eine erfolgreiche Aufforstung sein kann.

In der Schweiz kommt die Kompostanwendung für Aufforstungen im Mittelland nur auf relativ kleinen Flächen, z. B. bei schlechten Auenhöhen, in Frage. Für die Aufforstung in Berggebieten ist schon mit Erfolg versucht worden, Kompost direkt ins Pflanzloch zu geben. Beim grössten Teil 
der bestehenden Aufforstungsprojekte wird jedoch eine Vorbehandlung der Böden mit Kompost nicht notwendig sein.

\subsection{Gartenbau}

4.3 I Glashauskulturen und Treibbeete. Die Glashauskulturen gehören in den Niederlanden zu den besten Kompostabnehmern. Dies wird verständlich, wenn man bedenkt, dass in geheizten Glashäusern das ganze Jahr hindurch tropische Bedingungen herrschen, und daher der vorhandene Humusvorrat sehr rasch abgebaut wird; im Zusammenhang damit können Strukturverschlechterungen auftreten, die den Ertrag ernstlich gefährden. Man rechnet in den Niederlanden damit, dass pro Jahr etwa I50 $\mathrm{t}$ an organischen Bodendüngern angewandt werden müssen, nur um den Humusgehalt auf dem erwünschten Niveau von $5-6 \%$ zu erhalten.

In der Schweiz nimmt die Fläche unter Glas sehr rasch zu; sie beträgt heute etwa 200 ha. Es ist zu erwarten, dass sich hier ein weites Feld für den Kompostabsatz eröffnet.

In Treibbeeten wird die Verrottungswärme von Pferdemist zur Erwärmung des Bodens ausgenutzt. Pferdemist ist vielerorts nur schwer erhältlich, da die Pferdehaltung abgenommen hat. Es hat sich gezeigt, dass frisch aufbereiteter Müll (Frischkompost) für Treibbeete nicht nur dem Pferdemist ebenbürtig, sondern in mancher Hinsicht sogar überlegen ist, vor allem weil die Wärmeproduktion länger anhält. In den Niederlanden gibt es Kompostwerke, die die Hälfte ihrer Produktion für Treibbeete abgeben. Die Anlage der Trockenbeete mit Kompost wird im Prinzip gleich durchgeführt wie bei der Verwendung von Pferdemist.

4.32 Freilandkulturen. Im Erwerbsgartenbau ist eine ausgeprägte Tendenz zur Konzentration feststellbar, d. h. die kleinen Allerweltsgärtner verschwinden, und an ihre Stelle treten Grossgärtnereien, die auf wenige Produkte spezialisiert sind. Für den Kompostabsatz ist diese Entwicklung insofern günstig, als die Inhaber dieser Betriebe fachlich besser ausgebildet sind und auch schärfer kalkulieren; den Fragen der Bodendüngung und der Bodenbearbeitung dürfte daher in Zukunft eine grössere Aufmerksamkeit geschenkt werden als bisher. Da die Gartenbaubetriebe von jeher in grösserem Umfange organische Bodendünger zugekauft haben, werden überall dort die Aussichten für den Kompostabsatz günstig sein, wo dieser preislich mit anderen Materialien konkurrieren kann.

4.33 Neuanlage von Privatgärten, öffentlichen Anlagen usw. Die moderne Technik der Erdbewegung durch Raupenfahrzeuge bringt es mit sich, dass trotz aller Bemühungen und aller Sorgfalt beim Wiederaufbringen der 
Humuserde die Bodenstruktur nacher vielfach schlecht ist. Die Oberflächenerde ist bei neugeschaffenen Gartenanlagen meist mehr oder weniger stark mit Aushubmaterial durchsetzt, und der Untergrund durch die Fahrzeuge verdichtet. Massive Gaben von organischen Bodendüngern sind in vielen Fällen notwendig, um günstige Bedingungen für das Wachstum der Pflanzen zu schaffen. Gerade auf diesem Gebiet sind die Absatzmöglichkeiten für Kompost sehr gut, da dieser nicht nur preislich günstiger, sondern auch qualitativ wesentlich besser ist als Stallmist und Torf.

4.34 Privat- und Scbrebergärten, öffentliche Park- und Grünfläcben. In den privaten Gärten ist der Zustand der Böden meist mehr oder weniger schlecht, da die eigene Humusversorgung entweder ungenügend ist oder vollständig fehlt. Ferner ist durch die Bewässerung mit Leitungswasser der Boden häufig überkalkt.

Obwohl gesamthaft gesehen die Fläche dieser Gärten gross ist und daher auch grosse Kompostmengen abgesetzt werden könnten, ist es vom Kompostwerk aus gesehen nachteilig, kleine Mengen an sehr viele Einzelabnehmer zu liefern, d. h. einen besonderen Zubringerdienst, evtl. sogar eine Absackung, einzurichten. Für Schrebergärten, deren Besitzer in der Regel irgendwie organisiert sind, kommt eher der Verkauf en bloc in Frage, indem z. B. das Material an eine gemeinsame Abholstelle geliefert wird.

Die städtischen Gartenbauämter, denen der Unterhalt der öffentlichen Park- und Grünflächen zukommt, produzieren selber grössere Mengen an Gartenkompost aus den eigenen Abfällen; trotzdem gehören sie dort, wo bereits Kompostwerke bestehen, zu den besten Kompostabnehmern. Auch hier wäre zu überlegen, ob nicht die Aurbereitung der eigenen Abfälle (Fallaub usw.) nicht zweckmässiger in einem Kompostwerk durchgeführt würde.

\subsection{Weinbau}

Der Vorteil der Kompostanwendung im Weinbau liegt vor allem in der Möglichkeit einer Bekämpfung der Erosion an Steilhängen. Durch Kompostgaben von 100-200 t/ha in Abständen von 3-4 Jahren kann die Erosion weitgehend verhindert werden. Die Wirkung des Kompostes ist in dieser Hinsicht wesentlich besser als diejenige von Torf oder Stallmist, ganz abgesehen vom Preisunterschied.

In Versuchen auf der Halbinsel $\mathrm{Au}$, die von der Schweizerischen Weinbaufachschule durchgeführt wurden, konnte dazu eine eindeutige Verbesserung einiger physikalischer Bodeneigenschaften (Krümelstruktur, Porenvolumen, Versickerungsgeschwindigkeit usw.) festgestellt werden. Die mit 
Kompost behandelten Reben wiesen einen kräftigeren Blattwuchs auf und waren weniger chlorotisch als die unbehandelten. Dagegen konnte vorläufig noch kein Zusammenhang zwischen der Kompostanwendung und der Qualität und Quantität des Ertrages gefunden werden.

\subsection{Obstbau}

Im Ausland wird Kompost praktisch nur im Plantagenobstbau verwendet, jedoch dort mit sehr gutem Erfolg. In der Schweiz ist der Plantagenobstbau auf wenige Gebiete beschränkt (Wallis, Thurgau); Erfahrungen uiber die Kompostwirkung auf diesem Gebiet fehlen vorläufig noch.

\subsection{Strassenbau}

Bei der Neuanlage von Strassen steht meist genügend Humuserde für das Abdecken der Böschungen, Mittelstreifen usw. zur Verfügung; bei gewissen Teilstrecken dagegen und insbesondere auch bei kleineren Korrekturen an bestehenden Strassen ist dies jedoch oft nicht der Fall, und für die Begründung muss Humuserde von weit entfernt liegenden Bauplätzen herangeführt werden. Sofern ein Kompostwerk in der Nähe der Baustelle liegt, kann der Einsatz von Kompost wirtschaftlicher sein als das Heranbringen von Humuserde.

Ein besonderes Problem beim Strassenbau liegt darin, dass einerseits eine dichte Bewachsung und Durchwurzelung des Bodens zur Verhinderung der Wind- und Wassererosion erwünscht ist, anderseits aber ein zu üppiges Wachstum wegen der damit verbundenen Unterhaltsarbeiten nicht geschätzt wird. Die Aussichten für den Kompostabsatz sind daher auf diesem Gebiet so lange ungewiss, als nicht vermehrte Erfahrungen vorliegen.

\section{Die Beurteilung des potentiellen Kompostbedarfes bei der Projektierung einer Abfallbeseitigungsanlage}

Zur Ermittlung des potentiellen Kompostbedarfs, d. h. der effektiven Absatzmöglichkeiten für Kompost innerhalb eines bestimmten Gebietes, kann im Prinzip wie folgt vorgegangen werden:

a) Ermittlung der Gesamtfläche derjenigen Betriebe, die als Kompostabnebmer praktisch in Frage kommen. Für den Transport von Kompost gelten ähnliche Überlegungen wie für den Transport von Hausmüll; das Hauptabsatzgebiet ist daher begrenzt und liegt innerhalb eines Radius von $20-40 \mathrm{~km}$ um das Kompostwerk herum. Die Fläche der einzelnen Pflanzenbaubetriebe kann den amtlichen oder von Fachverbänden aufgestellten Statistiken entnommen werden. 
b) Ermittlung des absoluten Bedarfs an organischen Bodendüngern, umgerechnet auf Kompostäquivalente. Für die einzelnen Betriebsarten, insbesondere diejenigen des Intensivbaus, können Mittelwertefür den Bedarf an organischen Bodendüngern eingesetzt werden. Da nun gerade im Intensivbau Standort, Betriebsweise, Anteil der betriebseigenen Produktion an Bodendüngern und andere Faktoren sehr verschieden sind, müssen vorsichtshalber diese Mittelwerte eher niedrig angesetzt werden. Auf Grund in- und ausländischer Erfahrungen kann mit folgenden Werten gerechnet werden:

Sportplätze und andere Rasenanlagen

5 t/ha und Jahr

Öffentliche Grünanlagen, Haus- und Schrebergärten

Plantagenobstbau, Gemüsebau, private Baumschulen

Übriger Gartenbau

Forstgärten

Weinbau

Glashäuser

$20 \mathrm{t} /$ ha und Jahr

$25 \mathrm{t} / \mathrm{ha}$ und Jahr

Io t/ha und Jahr Ioo tha und Jahr $30 \mathrm{t} /$ ha und Jahr Ioo $t /$ ha und Jahr

Die übrigen Anwendungsgebiete wie

Strassenbau,

Aufforstungen,

Garten-Neuanlagen,

Spezielle Anwendungsgebiete in der Landwirtschaft

können nur sehr schwierig zahlenmässig erfasst werden; sie sind in einem gewissen Sinne als Absatzreserve zu betrachten.

c) Ermittlung des effektiven Kompostbedarfs. Es kann angenommen werden, dass etwa 10-20\% des absoluten Bedarfs auf Grund der Werte unter b) effektiv durch Kompost aus Siedlungsabfällen gedeckt werden wird; aus Sicherheitsgründen würden wir jedoch nur einen effektiven Bedarf von 10\% des Gesamtbedarfes einsetzen.

\section{Zusammenfassung}

Für die Verfahrenswahl bei der Projektierung einer Abfallbeseitigungsanlage spielt die Frage, ob Kompost in der näheren Umgebung abgesetzt werden kann oder nicht, eine wesentliche Rolle. Ferner ist bei Kompostwerken, die neu in Betrieb genommen wurden, mit einer gewissen Anlaufszeit zu rechnen, bis sich der Kompostabsatz eingespielt hat; während dieser Zeit ist eine gezielte Werbung notwendig, und es ist daher für den Betriebsleiter wichtig, $\mathrm{zu}$ wissen, wer als potentieller Abnehmer in erster Linie in Frage kommt. 
Die Anwendung von Kompost ist dort gegeben, wo man damit einen betrieblichen Vorteil irgendwelcher Art erzielen kann. Ob dies jedoch der Fall ist oder nicht, ist nicht immer einfach zu beurteilen; ganz abgesehen von den Schwierigkeiten bei der Anordnung, Durchführung und Auswertung von Versuchen, spielen sogar bei Fachleuten immer wieder Meinungen und Vorurteile weltanschaulich-dogmatischer Art mit hinein, die eine objektive Bewertung erschweren.

Die Aufgabe der vorliegenden Arbeit soll daher nicht darin bestehen, die Reihe der mehr theoretischen Arbeiten fortzusetzen, sondern es soll vielmehr versucht werden, rein empirisch auf Grund der vorliegenden Erfahrungen die Möglichkeiten des Komposteinsatzes im Pflanzenbau darzustellen.

Der Kompost ist in erster Linie Humuslieferant (Bodendünger); seine Nährstoffwirkung ist gering und daher sekundär. Seine Anwendung kommt dort in Betracht, wo man einerseits dem Humushaushalt besondere Aufmerksamkeit schenken muss, anderseits aber die Humusversorgung nicht auf betriebseigener Basis durchführen kann. Dies betrifft vor allem den Intensivbau, währenddem die Landwirtschaft aus wirtschaftlichen Gründen eher die Verbesserung der betriebseigenen Humusversorgung erstreben muss.

Die Humusstoffe beeinflussen die physikalischen und chemischen Eigenschaften des Bodens, d. h. sie wirken weniger direkt auf die Pflanze, sondern vielmehr indirekt durch Veränderung einiger Milieufaktoren. Sie tragen bei zur Schaffung eines ausgeglichenen Pflanzenmilieus und helfen die Pflanze vor extremen Stössen bewahren. Das Ziel der Kompostanwendung besteht also darin, optimale Bedingungen im Boden für das Pflanzenwachstum zu schaffen oder zu erhalten.

Die Kompostproduktion war früher darauf ausgerichtet, Humus für die Melioration extremer Böden zu erhalten. Heute hat sich das Schwergewicht aus den erwähnten Gründen immer mehr in den Intensivbau verlagert; der Kompost tritt hier in Konkurrenz zu den hergebrachten organischen Bodendüngern, vor allem dem Stallmist und dem Torf. Der Kompost aus Siedlungsabfällen ist dabei nicht nur für die meisten Anwendungsgebiete qualitativ überlegen, sondern auch preislich.

Für den Kompostabsatz kommen heute die folgenden Gebiete in Frage: r. Landwirtschaft: Nur in Ausnahmefällen und für besondere Zwecke, z. B. als Einstreu im Viehstall.

2. Forstwirtscbaft: In erster Linie in den Forstgärten, und ferner je nach den Verhältnissen auch für die Aufforstung. 
3. Gartenbau: Für alle Betriebszweige, je nach Standort, Betriebsweise usw. 4. Neuanlagen von Pripatgärten, öffentlichen Anlagen, Strassen usw.: Zur Verbesserung der durch die modernen Erdbewegungstechniken geschaffenen schlechten Böden, oder wenn nicht genügend Humuserde zum Abdecken des Aushubs zur Verfügung steht.

5. Weinbau: In erster Linie zur Bekämpfung der Erosion.

6. Obstbau: Vor allem im Plantagenobstbau und in den Baumschulen.

Zur Bestimmung des potentiellen Kompostbedarfs innerhalb eines transporttechnisch günstigen Absatzgebietes, d. h. zurErmittlung des mutmasslichen Kompostabsatzes bei der Projektierung einer Abfallbeseitigungsanlage, können die Fläche derjenigen Betriebe, die als Abnehmer in Frage kommen, auf Grund der Statistik festgestellt und dann, mit Hilfe von Richtzahlen für die einzelnen Anwendungsgebiete, die effektiven Absatzmöglichkeiten errechnet werden. 